Conclusions Concordance between preferred and actual treatment is high in older people who prefer treatment, and lower in people who prefer no treatment. Yet, making preferences for forgoing treatment known is useful as it increases the chance of treatments being forgone in those who wish so.

\title{
17 PATIENT PREFERENCES AND ACTUAL TREATMENT IN OLDER PEOPLE AT THE END OF LIFE. A MORTALITY FOLLOW-BACK STUDY
}

\author{
H Roeline, ${ }^{1}$ W Pasman, ${ }^{1}$ Pam J Kaspers, ${ }^{1}$ Dorly J H Deeg, ${ }^{2}$ Bregje \\ D Onwuteaka-Philipsen' ${ }^{1}$ VU University Medical Center, EMGO Institute for \\ Health and Care Research, Department of Public and Occupational Health, \\ Expertise Center for Palliative Care, van der Boechorststraat 7, 1081 BT, \\ Amsterdam, The Netherlands; ${ }^{2}$ VU University Medical Center, EMGO Institute \\ for Health and Care Research, Department of Epidemiology and Biostatistics, \\ Longitudinal Aging Study Amsterdam (LASA), van der Boechorststraat 7, \\ 1081 BT, Amsterdam, The Netherlands
}

10.1136/bmjspcare-2013-000491.17

Background Respecting patients' preferences is seen as good palliative care.

Aim Studying actual treatments in relation to preferences for starting or forgoing treatment of older people at the end of life.

Methods Mortality follow-back study among relatives of deceased older people (2006-2009) of two cohorts representative for the older Dutch population $(\mathrm{n}=168)$ and for people with an advance directive $(n=184)$. Preferences and actual treatment were studied for each of four treatments: starting or forgoing resuscitation, artificial nutrition/hydration, antibiotics, and artificial respiration.

Results In most patients who preferred receiving treatment this preference was followed $(88 \%$ to $100 \%$ for different situations). In about half of the patients who preferred that a treatment would be forgone, the preference was followed (except artificial respiration: 12\% concordance). The majority of people for whom no preference was known received treatment (59\% to 79\% for different situations). People with a known preference for receiving a specific treatment (as opposed to forgoing that treatment) had a higher chance of preference being followed (OR 7.4). People with a preference for forgoing a treatment had higher odds (OR 6.3), and people with a preference for starting a treatment had lower odds (OR 0.28) of treatment being forgone compared to people having no known preference. 